\title{
Evaluating the Pre-Emptive Analgesic Effect of Single Dose Oral Paracetamol in Ureteroscopic Lithotripsy Procedures- A Double Blinded Randomised Controlled Study
}

\author{
Senthil Kumar Kaliannan¹, Pavan Kumar Dammalapati², Khaja Mohideen Sherfudden³ \\ ${ }^{1}$ Consultant Anaesthesiologist, Department of Anaesthesiology, Kauvery Hospitals, Trichy, Tamilnadu, India. \\ ${ }^{2}$ Consultant Anaesthesiologist, Department of Anaesthesiology, Kauvery Hospitals, Trichy, Tamilnadu, India. \\ ${ }^{3}$ Consultant Anaesthesiologist, Department of Anaesthesiology, Kauvery Hospitals, Trichy, Tamilnadu, India.
}

\section{ABSTRACT}

\section{BACKGROUND}

Pain after endoscopic urological surgeries differs from other open surgeries as the pain is mainly due to bladder spasms or catheter related. Pre-emptive analgesia decreases the analgesic requirements perioperatively. The purpose of this study is to evaluate the pre-emptive analgesic effect of oral paracetamol $1000 \mathrm{mg}$ in patients undergoing ureteroscopic lithotripsy procedures.

\section{METHODS}

This is a double blinded randomised controlled study. The study included 60 ASA I II patients admitted for ureteroscopic lithotripsy procedures. Patients were randomly allocated into two equal groups: group P received oral Paracetamol $1 \mathrm{gm}$ and group $C$ received oral Vitamin C $500 \mathrm{mg}$ as placebo, 1 hour before surgery. The intensity of postoperative pain was measured with 0-10-point Numerical Rating Scale (NRS). Rescue analgesia was given if patients had NRS scores of 4 or more. Time to first rescue analgesia was noted. Statistical analysis was done with Chi square and independent sample t test.

\section{RESULTS}

At $3^{\text {rd }}, 4^{\text {th }}$ and $5^{\text {th }}$ hour, the pain scores were low in paracetamol group compared to placebo group. Time to rescue analgesia was more in paracetamol group compared to placebo group. Both the parameters were statistically significant.

\section{CONCLUSIONS}

Oral paracetamol $1 \mathrm{gm}$ used as a pre-emptive analgesia in patients undergoing ureteroscopic lithotripsy procedures, provides excellent analgesia compared to control group with no side effects.

\section{KEY WORDS}

Pre-Emptive Analgesia, Paracetamol, Endoscopic Urological Surgeries

\author{
Corresponding Author: \\ Pavan Kumar Dammalapati, \\ Consultant Anaesthesiologist, \\ Department of Anaesthesiology, \\ Kauvery Hospitals, Trichy, \\ Tamilnadu, India. \\ E-mail: pavanmedico81@yahoo.com
}

DOI: $10.14260 /$ jemds $/ 2019 / 626$

Financial or Other Competing Interests: None.

How to Cite This Article:

Kaliannan SK, Dammalapati PK, Sherfudeen KM. Evaluating the preemptive analgesic effect of single dose oral paracetamol in ureteroscopic lithotripsy procedures- a double blinded randomised controlled study. J. Evolution Med. Dent. Sci. 2019;8(38):2881-2885, DOI: $10.14260 /$ jemds $/ 2019 / 626$

Submission 31-07-2019,

Peer Review 08-09-2019,

Acceptance 14-09-2019,

Published 23-09-2019. 


\section{BACKGROUND}

Pain is a protective phenomenon even though it is quite unpleasant. It is a common clinical problem encountered and the incidence in post-surgical patients is almost $75 \%$.(1) Preemptive analgesia reduces magnitude and duration of pain. Pre-emptive analgesia prevents the establishment of altered processing of afferent input. This concept of anti nociception was started by Woolf way back in 1983.(2)

Paracetamol is a potent anti-pyretic and analgesic but is a weak anti-inflammatory. Mechanism of action is central cyclooxygenase inhibition and an indirect influence on the serotonergic system. It has a good safety profile and easily passes through the blood brain barrier.(3) There are many studies, which compared the analgesic and anti-inflammatory effect of different analgesics with paracetamol which did not show any advantage over paracetamol.(4) The advantage of paracetamol over other analgesics is its safety and tolerability. Use of non-opioid analgesic drugs like paracetamol in perioperative period reduces the intraoperative use of opioids.

Pain after endoscopic urological surgeries differ from other open surgeries as the pain is mainly due to bladder spasms and catheter related.(5)

The intra venous route of administration is associated with higher incidence of anaphylactic reactions. The cost difference between an intravenous paracetamol preparation to the oral drug is significant. In case of paracetamol, the oral drug is 200 times not only cheaper but also equally efficacious and hence it reduces the financial burden to the patient. There are very few studies about using oral paracetamol as a pre-emptive analgesia on postoperative pain.

We wanted to assess the pre-emptive analgesic efficacy of $1 \mathrm{gm}$ of oral paracetamol in patients undergoing ureteroscopic lithotripsy procedures under spinal anaesthesia.

\section{METHODS}

This prospective, randomised, control study was conducted after getting institutional ethical committee approval and informed written consent from patient. Sample size was calculated based on the assumption that there would be $20 \%$ or more difference in time to first rescue analgesia calculated postoperatively. This required 30 patients in each group for the results to be significant (with alpha of 0.05 and power of $100 \%)$. For this study, only institutional ethics committee approval was obtained and clinical trial registration in CTRI (Clinical Trial Registry - India) was not done before the start of study. Patients aged between 20 to 60 years of ASA I and ASA II status undergoing endoscopic urological surgeries were included in the study.

Patients were randomly assigned to two treatment groups (Group C and Group P) with the help of a computergenerated table of random numbers. In Group C (Control group) - Oral Vitamin C $500 \mathrm{mg}$ is given one hour before surgery and in Group P (Paracetamol group)- Oral Paracetamol $1 \mathrm{gm}$ is also given one hour before surgery. Pre anaesthetic assessment done and strict Nil Per Oral orders followed. In the operating theatre, standard monitors connected. Spinal anaesthesia is given with $0.5 \%$ Bupivacaine with dextrose in the dose of $0.3 \mathrm{mg} / \mathrm{kg}$ given at the level of L3 and L4 interspace with 26 gauze Quincke spinal needle.

Sedatives and analgesics were not used intra-operatively. Haemodynamics (Mean arterial pressure and pulse rate) were recorded intra-operatively and postoperatively till the patient complains of pain. A change of $20 \%$ was considered as significant. Numeric Rating Scale (NRS) is used for pain assessment at 5-minute interval till first rescue analgesic is given. Tramadol $50 \mathrm{mg}$ was given intravenously as rescue analgesic if NRS > 4. Nausea and vomiting in the postoperative period were also recorded.

\section{Statistical Analysis}

Statistical analysis was done using SPSS software version 20, Excel Data Plugin. Data is represented as mean \pm standard deviation. Chi-square test (For sex and ASA status) and independent sample $\mathrm{t}$ tests were used. $\mathrm{P}<0.05$ was considered significant.

\section{RESULTS}

\begin{tabular}{|c|c|c|c|}
\hline Variable & Group C & Group P & p Value \\
\hline Age (years) & $45.53 \pm 14.0$ & $45.67 \pm 15.8$ & 0.973 \\
\hline Sex & $14 / 16$ & $18 / 12$ & 0.438 \\
\hline ASA status (1/2) & $20 / 20$ & $19 / 11$ & 1.0 \\
\hline Weight (Kg) & $66.80 \pm 9.9$ & $65.50 \pm 13.4$ & 0.671 \\
\hline Duration of surgery (minutes) & $66.67 \pm 21.9$ & $68.83 \pm 21.9$ & 0.703 \\
\hline Table 1. Demographic Characteristics of the Patients and \\
Duration of Surgery \\
\hline
\end{tabular}

Comparison of the two study groups for age, weight, gender ratio and ASA status revealed no statistically significant intergroup difference $(p>0.05)$ [Table 1]. Duration of surgery was also comparable between two groups [Table 1].

\begin{tabular}{|c|c|c|c|}
\hline Variable & Group C & Group P & p Value \\
\hline Time to first rescue analgesia (minutes) & $211.67 \pm 33.6$ & $290.33 \pm 40.0$ & 0.00 \\
\hline \multicolumn{3}{|c|}{ Table 2. Time to First Rescue Analgesia } \\
\hline
\end{tabular}

Rescue Analgesia was administered much later (290.33 \pm 40.0) in the Paracetamol group than the Control group $(211.67 \pm 33.6)$ and the difference in means of the two groups was statistically significant [Table 2].

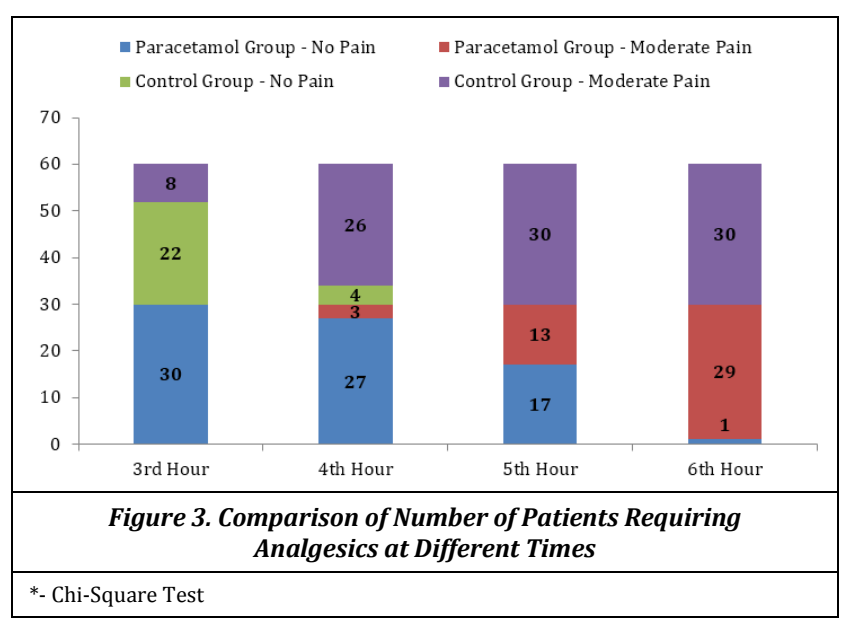


The Mean Arterial Pressure and the mean pulse rate are within normal limits in both the Paracetamol group and the Control group and does not have statistically significant difference throughout the postoperative period.

It was observed that none of the patients among both the study groups experienced pain in the first and second Postoperative hour. On the basis of Numeric Rating Scale measured at $3^{\text {rd }}$ hour it can be seen that none of the patients belonging to Paracetamol group complained of pain but about one fifth $(26.7 \%)$ of patients in the Control group complained of mild to moderate pain ( $p$ value -0.05 ). In the $4^{\text {th }}$ hour it was observed that most (87.7\%) of the patients in the Control group complained of pain wherein only 10 percent of the Paracetamol group needed rescue analgesia ( $p$ value -0.00 ). In the $5^{\text {th }}$ hour all the patients in the Control group (100\%) complained of pain whilst less than half $(43.4 \%)$ of the patients in the Paracetamol group needed rescue analgesia ( $p$ value -0.00$)$. In the $6^{\text {th }}$ hour almost all patients complained of pain signifying the need for rescue analgesia in all patients ( $p$ value -1.0). The association between the study groups and the perception of pain was found to be statistically significant at all times except at $6^{\text {th }}$ hour.

\begin{tabular}{|c|c|c|c|c|c|c|}
\hline \multicolumn{2}{|c|}{ Group } & $\mathbf{N}$ & $\begin{array}{l}\text { Mean } \\
\text { Rank }\end{array}$ & $\begin{array}{c}\text { Sum of } \\
\text { Ranks }\end{array}$ & $\begin{array}{c}\text { Mann-Whitney } \\
\text { U Test }\end{array}$ & $\begin{array}{c}\text { p- } \\
\text { Value }\end{array}$ \\
\hline \multirow{2}{*}{ NRS0 } & Paracetamol & 30 & 30.50 & 915.00 & \multirow{2}{*}{450} & \multirow{2}{*}{1.00} \\
\hline & Control & 30 & 30.50 & 915.00 & & \\
\hline \multirow{2}{*}{ NRS1 } & Paracetamol & 30 & 30.50 & 915.00 & \multirow[b]{2}{*}{450} & \multirow{2}{*}{1.00} \\
\hline & Control & 30 & 30.50 & 915.00 & & \\
\hline \multirow{2}{*}{ NRS2 } & Paracetamol & 30 & 30.50 & 915.00 & \multirow{2}{*}{450} & \multirow{2}{*}{1.00} \\
\hline & Control & 30 & 30.50 & 915.00 & & \\
\hline \multirow{2}{*}{ NRS3 } & Paracetamol & 30 & 26.50 & 795.00 & \multirow{2}{*}{330} & \multirow{2}{*}{0.003} \\
\hline & Control & 30 & 34.50 & 1035.00 & & \\
\hline \multirow{2}{*}{ NRS4 } & Paracetamol & 30 & 18.28 & 548.50 & \multirow{2}{*}{83.5} & \multirow{2}{*}{0.00} \\
\hline & Control & 30 & 42.72 & 1281.50 & & \\
\hline \multirow{2}{*}{ NRS5 } & Paracetamol & 30 & 17.98 & 539.50 & \multirow{2}{*}{74.5} & \multirow{2}{*}{0.00} \\
\hline & Control & 30 & 43.02 & 1290.50 & & \\
\hline \multirow{2}{*}{ NRS6 } & Paracetamol & 30 & 21.77 & 653.00 & \multirow{2}{*}{188} & \multirow{2}{*}{0.00} \\
\hline & Control & 30 & 39.23 & 1177.00 & & \\
\hline
\end{tabular}

Table 3. Distribution of Mean Ranks of the Study Groups with Respect to the Pain Perception Based on Numeric Rating Scale

The above table shows the mean ranks of the Pain perception scale namely the Numeric Rating Scale of the patients belonging to both the Paracetamol group and Control group seen at one-hour interval in the postoperative period. It was observed that there was no difference in the mean ranks in the first 2 hours because none experienced any degree of pain. From the $3^{\text {rd }}$ hour onwards it was appreciated that the mean ranks of Paracetamol group were consistently lower than the Control group at $3^{\text {rd }}$ hour (26.50 against $34.50), 4^{\text {th }}$ hour (18.28 against 42.72$), 5^{\text {th }}$ hour (17.98 against 43.02) and $6^{\text {th }}$ hour (21.77 against 39.23$)$. The difference in mean ranks of the study groups was significantly associated between the Paracetamol group and the Control group. As such it can be seen that the patients in the Paracetamol group perceived less pain than the Control group.

From the below table, it is evident that nearly three fourths $(73.3 \%)$ of the patients in the Paracetamol group were free of complications whereas less than one third (30\%) of the patients in the Control group were complications free $(p=0.02)$. As the symptoms of nausea and vomiting are generally related to be due to the administration of Tramadol given for rescue analgesia, increased complications seen in the Control group may be ascribed to early or repeated administration of rescue analgesia in patients in the control group because of poor pain relief.

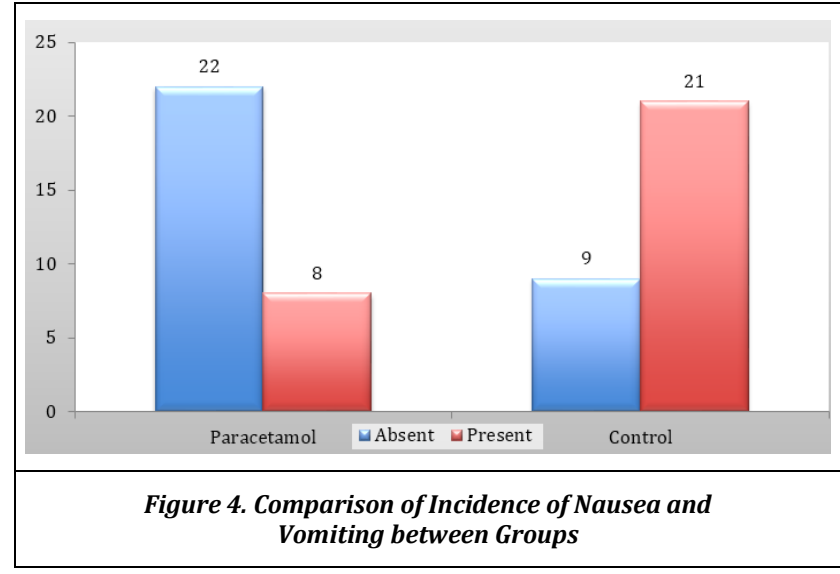

\section{DISCUSSION}

If pain relief is adequate; there will be reduced patient's anxiety, morbidity, costs of care and duration of hospitalization.(6) Pre-emptive analgesia attenuates the pain by blocking the nervous system's usual response to pain.

Endoscopic urological surgeries like Ureteroscopic Lithotripsy (URSL), Endoscopic incision of ureterocele (EIU), Transurethral resection of bladder tumour (TURBT), transurethral resection of prostrate(TURP), percutaneous nephro lithotripsy (PCNL) carries mild to moderate pain in the postoperative period.(7) All these patients will be invariably catheterized and pain associated with movement and traction of catheter is in addition to the pain at the operative site.(5) Most of these surgeries are associated with little tissue damage and hence chances of severe inflammatory response are minimal. We decided to select URSL patients for our study.

In this study we compared postoperative pain score in two groups, ie., paracetamol and control. The results showed that the pain scores in the PACU were lower in the paracetamol group than in the control group. At $3 \mathrm{hrs}$ after surgery $8 / 30$ patients complained of moderate pain (NRS $4-$ 7) in the placebo group whereas no patients in the paracetamol group complained of pain. $(p=0.05)$ At 4 hrs after surgery, $26 / 30$ patients in the control group complained of moderate pain whereas only 3 patients in the paracetamol group complained of pain; which is very significant $(p=0.00)$. At 5 hours interval, 13 patients in paracetamol group and all the patients in the control group complained of moderate pain $(\mathrm{p}=0.00)$. Finally, at 6 hours postoperatively, 29 patients in the paracetamol group and all patients in the control group experienced pain $(\mathrm{p}=1.00)$ which are statistically equal. Hence it can be concluded that Oral paracetamol is more efficacious than placebo when administered preoperatively for pain management in endoscopic urological surgeries.

Kaluzny et al reported that preoperative oral administration of $1 \mathrm{gm}$ acetaminophen was effective, convenient, safe and cost effective in reducing the pain during and following the operation, in phacoemulsification performed using topical anaesthesia, ${ }^{(8)}$ which is very much comparable to our study. However, Bennie et al demonstrated in their study that high dose of acetaminophen had similar analgesic effect as ibuprofen after myringotomy in paediatric patients. In addition, there was no significant 
difference between the analgesic effects of acetaminophen, ibuprofen and placebo after myringotomy.(9)

The time to rescue analgesia in the paracetamol group was an average of $290.33 \pm 40.0$ minutes whereas in the control group it was $211.67 \pm 33.6$ minutes $(\mathrm{p}<0.00)$, which is very significant. McNicol et al., in their study of randomized control trial, compared Intravenous paracetamol to the placebo group for acute postoperative pain and concluded that the I.V paracetamol is superior. This data is correlating with the data published by McNicol et al., ${ }^{(10)}$ where they have a randomized controlled trial which used single dose I.V. Paracetamol for acute postoperative pain relief. Propacetamol and paracetamol were superior to placebo over both 4 and $6 \mathrm{~h}$, the proportion of patients with at least $50 \%$ pain relief appears to decrease at $6 \mathrm{~h}$ in both active groups (and in the placebo groups). Our study also correlated with the study by Remy et al.,(11) where among secondary outcomes, data related to rescue medication demonstrated that fewer patients receiving propacetamol or paracetamol required rescue analgesia in the 4-6 $\mathrm{h}$ time period than those receiving placebo, and those that did require rescue analgesia waited longer before requesting it than those receiving placebo. In the majority of studies comparing opioid consumption, a PCA was used. Also, the study, which was done by Reuben et al., (12) showed similar results as our study. These results are comparable to the studies done by Seymour et al., with various analgesics. $(13,14)$

When comparing the adverse effects i.e., nausea and vomiting between the two groups there are 8 patients $(26.7 \%)$ who are having nausea and vomiting in the paracetamol group whereas there are 21 patients (70.0\%) having the same in control group. The results are not correlated with the results of McNicol et al.,(10) where the incidence of adverse events that could be considered to be opioid-induced have found no difference in side-effects, despite the reported reduction in opioid requirements. In contrast, meta-analyses of NSAIDs used in combination with PCA by Elia et al.,(15) demonstrate a relative reduction in postoperative nausea and vomiting by $30 \%$, nausea alone by $12 \%$, vomiting alone by $32 \%$, and sedation by $29 \%$ which is in comparison to our study. Also, our study is comparable to the study done by Moon et al., (16) who demonstrated that premedication with acetaminophen reduced hydromorphone consumption and opioid-related side effect in patients undergoing abdominal hysterectomy.

The effects of pre-emptive analgesia in postoperative pain relief in ophthalmic surgeries were showed in some studies.(17) The role of pre-emptive use of acetaminophen in reducing postoperative pain was shown in paediatric tonsillectomy patients(18) and paediatric patients undergoing bilateral myringotomy. $(19,20)$ Our data are comparable with the results of these studies.

Our study showed that Oral Paracetamol one gram has longer duration of analgesia than placebo. Similar studies were done by Moller et al.,(21) where they compared the efficacy and safety of propacetamol $2 \mathrm{~g}$ bolus or infusion with oral acetaminophen $1 \mathrm{~g}$ or placebo for analgesia after third molar surgery in patients with moderate to severe pain. They concluded that the onset of analgesia after I.V. propacetamol was shorter ( $3 \mathrm{~min}$ for bolus administration, $5 \mathrm{~min}$ for 15min infusion) than oral acetaminophen (11 min). The duration of analgesia was significantly longer after all three active treatments than after placebo. The duration of analgesia was significantly longer after oral acetaminophen (278 min) than after bolus Propacetamol (180 min), for infusion propacetamol (171 $\mathrm{min}$ ) and $68 \mathrm{~min}$ for Placebo. In our study the rescue analgesia duration was $290.33 \pm 40.0$, which is very much comparable to the above study.

The cost of 1 vial of Injection Paracetamol is around Rs. 300/- (Rupees Three hundred only) but the cost of 1 gram of oral tablet paracetamol is around Rs. 2/- (Rupees Two Only). Such a large variation exists between the costs of the two drugs. Hence wherever is possible and if there are no contraindications to oral usage, oral tablet paracetamol can be used.

\section{Limitations}

This study is conducted on patients undergoing regional anaesthesia, so the hemodynamic variations/ stability pertaining to the use of paracetamol compared to placebo cannot be assessed perioperatively. The onset of analgesia by the study drug- paracetamol also cannot be mentioned as it would be masked by the residual analgesic effects of the regional anaesthesia.

\section{CONCLUSIONS}

Oral paracetamol $1 \mathrm{gm}$ used as a pre-emptive analgesia in patients undergoing ureteroscopic lithotripsy procedures, provides excellent analgesia without side effects as compared to control group.

\section{REFERENCES}

[1] Guyton AC, Hall JE. Textbook of medical physiology. 11 $1^{\text {th }}$ edn. Pennsylvania: Elsevier Inc., 2006.

[2] Cohen FL. Postsurgical pain relief: patients' status and nurses' medication choices. Pain 1980;9(2):265-74.

[3] Woolf CJ. Evidence for a central component of post injury pain hypersensitivity. Nature 1983;306(5944):686-8.

[4] Kissin I. Pre-emptive analgesia. Anesthesiology 2000;93(4):1138-43.

[5] Kara C, Resorlu B, Cicekbilek I, et al. Analgesic efficacy and safety of non-steroidal anti-inflammatory drugs after transurethral resection of prostate. Int Braz J Urol 2010;36(1):49-54.

[6] Shahriari M, Golshan A, Alimohammadi N, et al. Effects of pain management program on the length of stay of patients with decreased level of consciousness: a clinical trial. Iran J Nurs Midwifery Res 2015;20(4):502-7.

[7] Heid F, Jage J. The treatment of pain in urology. BJU Int 2002;90(5):481-8.

[8] Kaluzny BJ, Kazmierczak K, Laudencka A, et al. Oral acetaminophen (paracetamol) for additional analgesia in phacoemulsification cataract surgery performed using topical anesthesia randomized double-masked placebo-controlled trial. J Cataract Refract Surg 2010;36(3):402-6. 
[9] Bennie RE, Boehringer LA, McMahon S, et al. Postoperative analgesia with preoperative oral ibuprofen or acetaminophen in children undergoing myringotomy. Paediatr Anaesth 1997;7(5):399-403.

[10] McNicol ED, Tzortzopoulou A, Cepeda MS, et al. Singledose intravenous paracetamol or proparacetamol for prevention or treatment of postoperative pain: a systematic review and meta-analysis. $\mathrm{Br} \mathrm{J}$ Anaesth 2011;106(6):764-75.

[11] Remy C, Marret E, Bonnet F. Effects of acetaminophen on morphine side-effects and consumption after major surgery: meta-analysis of randomized controlled trials. British Journal of Anaesthesia 2005;94(4):505-13.

[12] Reuben SS, Bhopatkar S, Maciolek H, et al. The preemptive analgesic effect of rofecoxib after ambulatory arthroscopic knee surgery. Anesth Analg 2002;94(1):559.

[13] Seymour RA, Kelly PJ, Hawkesford JE. The efficacy of ketoprofen and paracetamol (acetaminophen) in postoperative pain after third molar surgery. Br J Clin Pharmacol 1996;41(6):581-5.

[14] Seymour RA, Hawkesford JE, Weldon M, et al. An evaluation of different dosages and preparations of ibuprofen in post-operative dental pain after third molar surgery. Br J Clin Pharmacol 1991;31(1):83-7.

[15] Elia N, Lysakowski C, Tramer MR. Does multimodal analgesia with acetaminophen, non-steroidal antiinflammatory drugs, or selective cyclooxygenase- 2inhibitors and patient-controlled analgesia morphine offer advantages over morphine alone? Meta-analyses of randomized trials. Anesthesiology 2005;103(6):1296304.

[16] Moon YE, Lee YK, Lee J, et al. The effects of preoperative intravenous acetaminophen in patients undergoing abdominal hysterectomy. Arch Gynecol Obstet 2011;284(6):1455-60.

[17] Mahfouz AK, Nabawi KS. Pre-emptive analgesia in rhegmatogenous retinal detachment surgery: is it effective? Retina 2002;22(5):602-6.

[18] Romej M, Voepel-Lewis T, Merkel SI, et al. Effect of preemptive acetaminophen on postoperative pain scores and oral fluid intake in pediatric tonsillectomy patients. AANA J 1996;64(6):535-40.

[19] Watcha MF, Ramirez-Ruiz M, White PF, et al. Perioperative effects of oral ketorolac and acetaminophen in children undergoing bilateral myringotomy. Can J Anaesth 1992;39(7):649-54.

[20] Ragg P, Davidson A. Comparison of the efficacy of paracetamol versus paracetamol, codeine and promethazine (Painstop) for premedication and analgesia for myringotomy in children. Anaesth Intensive Care 1997;25(1):29-32.

[21] Moller PL, Sindet-Pedersen S, Petersen CT, et al. Onset of acetaminophen analgesia: comparison of oral and intravenous routes after third molar surgery. British Journal of Anaesthesia 2005;94(5):642-8. 